\title{
MANET Reactive Protocols-Tutorial Review
}

\author{
Jahangir Khan \\ Graduate Institute of science and \\ Engineering, PAF-KIET, PAF Base \\ Korangi Creek \\ Karachi 75190, Pakistan.
}

\begin{abstract}
In the competition of this modern technology the aim of Mobile Ad Hoc networking is to provide efficient communication in wireless technology by adopting routing functionality in mobile nodes. The main aim behind the developing of ad hoc networking is multi-hop relaying. Wireless Ad hoc networks or infrastructure less networks are very easy to establish by using radio waves as transmitting medium without the requirements of any other equipment or infrastructure. In such a network mobile nodes can be moved and organized freely in an arbitrary way. But still the fast growing technology needs attention in many areas such as routing, bandwidth, security, power consumption, collisions, simulations, and topology control due to moving nodes. In this tutorial I have proposed reactive(on-demand) Routing protocols for accurate and best performance data delivery in ad-hoc networks with deferent frequencies based on OPNET simulator. The objective of this paper is to extend and evaluate proposed routing protocols to be suitable for mobile ad hoc networks in autonomous system.
\end{abstract}

\section{General Terms: AODV, DSR, MANET, OPNET.}

Keywords: Ad-Hoc Networks, Performance, efficiency, Data fraction.

\section{INTRODUCTION}

In the fast world of growing technology the advancement in computers and mobile wireless communication technology have increasingly used applications in every area of life. Most of the technology operates in the traditional Internet Protocol (IP) suite. In the competition of this modern technology the aim of Mobile Ad-Hoc networking is to provide efficient communication in wireless technology by adopting routing functionality in mobile nodes. The main aim behind the developing of ad hoc networking is multi-hop relaying. Wireless Ad-Hoc networks or infrastructure less networks are very easy to establish by using radio waves as transmitting medium without the requirements of any other equipment or infrastructure. In such a network mobile nodes can be moved and organized freely in an arbitrary way. This dynamic connectivity of nodes allow mobile ad hoc network to be organized any where any time. Loads of research work and efforts have been done since last decade to provide support and solution to different problems and challenges related to mobile ad hoc networks. But still the fast growing technology needs attention in many areas such as routing, bandwidth, security, power consumption, collisions, simulations, and topology control due to moving nodes. For best QOS I have proposed on-demand routing protocols in hard real time basis for timely delivery of data packets in an autonomous system. As clear form the name on- demand routing also called reactive routing protocols, these protocols do not exchange routing information periodically but use flooding method to obtain information when required for a node to send data packet. The host node which needs to transmit packets to destination in network, broadcasts a route request to all nodes in network. The host node will be waiting for reply of the nodes in the network to provide a path to destination before transmitting packets. Reactive protocol performs two main functions in Ad hoc network routing, i.e. route discovery and route maintenance. The route discovery function of protocol is responsible for detecting of new route to forward packets when required by a node. Route maintenance function of reactive protocol is responsible for detecting of broken links and repairing of an existing route. The route discovered by global search process are then stored for future use until they become invalid and deleted because of link failure or topological changes. The route request and selection process leads to significant delay in initial setup of transmitting packets. Therefore real time application using on-demand routing protocols requires transmission of significant amount of control overhead due to global or flooding search method. Reactive protocols are bandwidth efficient and reduce storage space as it does not need to keep unnecessary network topology information up-to-date. The common reactive routing protocols used by MANET are DSR, AODV, TORA, ABR, SSA, LAR, FORP, LMR, ROAM and PLBR.

The maturity of wireless mobile technologies and the evolution of different applications provide a reason for the introduction of QoS in wireless ad hoc networks. The goal of QoS routing in MANET is to select routes with sufficient resources for data packets with QoS requirements to increase possibility that network will be capable of supporting and maintaining them. Here I proposed two reactive routing protocols along with simulation based results for efficient and accurate data delivery on timely basis using OPNET simulator V.12. In order to judge the performance of on-demand routing protocols one needs to know different metrics to deliver a greater percentage of originated data packets where there is node mobility occur. Both Dynamic source Routing (DSR) and Ad-hoc on-demand Distance Vector routing protocol (AODV) are ondemand protocols whose basic characteristic is demonstrated in the shape of its overhead. Some simulation parameters and results are illustrated here for optimization techniques to achieved and improve performance and to reduce overhead.

\section{PARAMETERS}

Simulation parameters are: Simulation time 300s, Maximum pause time 0 and 200s, Transmission rates 2, 5, and 10 packets/s, Packet size 512 bytes, Movement space 4000m x 3000m, Number 
of wireless nodes 6 and 25 nodes. This precedes for the results paradigms.

\section{IMPACT OF QOS IN AD-HOC PROTOCOLS}

The maturity of wireless mobile technologies and the evolution of different applications provide a reason for the introduction of QoS in wireless ad hoc networks. The goal of QoS routing in MANET is to select routes with sufficient resources for data packets with QoS requirements to increase possibility that network will be capable of supporting and maintaining them. Here I proposed two reactive routing protocols along with simulation based results for efficient and accurate data delivery on timely basis using OPNET simulator V.12. In order to judge the performance of on-demand routing protocols one needs to know different metrics to deliver a greater percentage of originated data packets where there is node mobility occur. Both Dynamic source Routing (DSR) and Ad-hoc on-demand Distance Vector routing protocol (AODV) are ondemand protocols whose basic characteristic is demonstrated in the shape of its overhead. Some simulation parameters and results are illustrated here for optimization techniques to achieved and improve performance and to reduce overhead.

\section{SIMULATION RESULTS OF AODV AND DSR ROUTING PROTOCOLS}

In 6-25 nodes scenario for AODV, the Traffic sent in bits is higher as compared to the packets received. Once route is established routing traffic flows in a continuous way along data between wireless nodes of ad hoc network. Each hop in discovered route adds some routing traffic to data. Traffic received is combination of all hops occurs in route from source to destination. Therefore routing traffic received is very high as compared to traffic sent. The maximum traffic sent in bits per second is between 1300 and 1400, where in packets per second are between 3 and 3.5. Most of the time during simulation period the routing traffic is $0 \mathrm{bits} / \mathrm{s}$ and packets/s. On the other side maximum traffic received at destination node is much higher which is between 2800 and 3000 bits per second and is 7 packets per second which indicate that the traffic signal is very high in first and second time route establishment as in figure 1. and Figure 2 respectively.

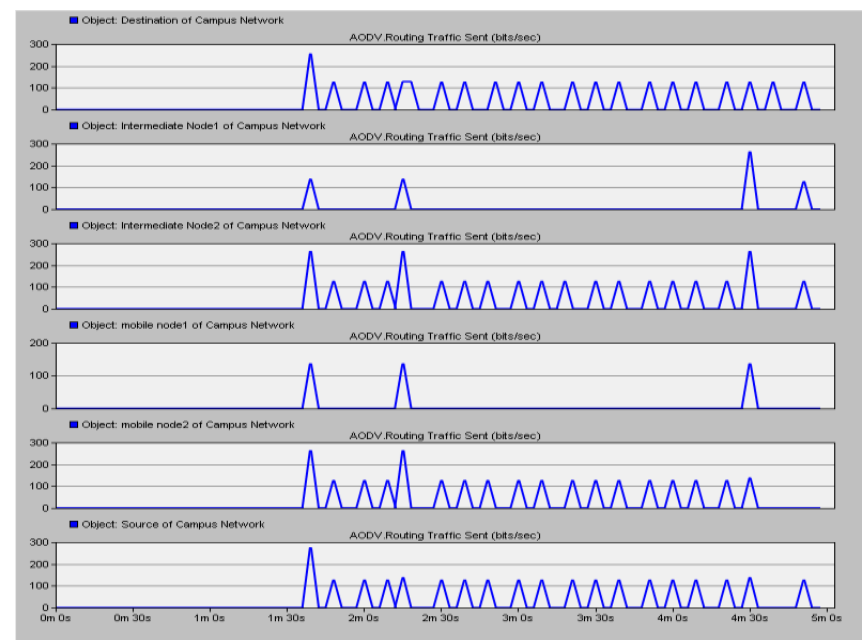

Figure1. Total AODV routing traffic sent

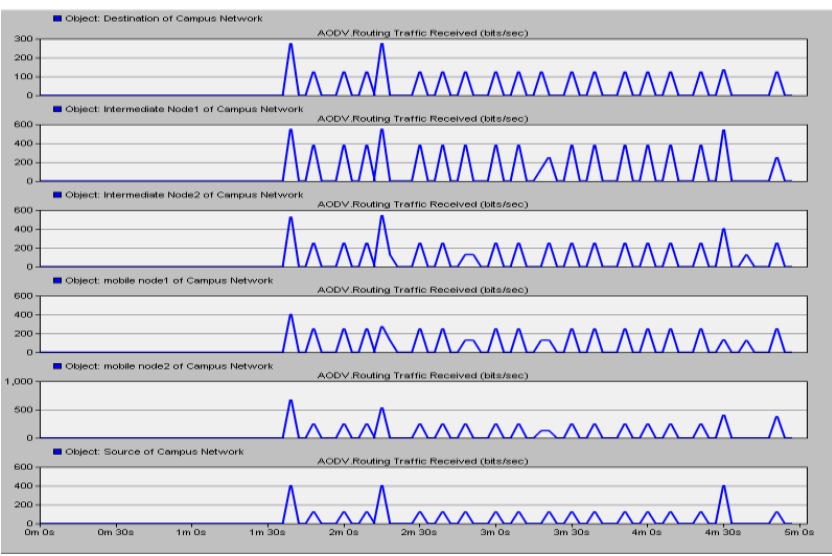

Figure 2. Total AODV routing traffic Received.

As discussed earlier that the selected route consist of 6-25 nodes scenario, in which the four nodes have strong interactions, i.e. source, mobile node 2 , intermediate node 2 , and destination. The high level of traffic is sent by intermediate node 2 and mobile node 2 during simulation time. The source node sent a bit high routing traffic of $300 \mathrm{bits} / \mathrm{sec}$ once during first route discovery process. The intermediate node 2 sends extra high traffic of 290 bits/sec each time the route discovery process occurs. The detail analysis of the graph shows that routing data generated at source node and destination node is nearly the same at. The intermediate nodes show the high traffic signals because of Hello messages to keep alive the next neighbor nodes. The routing traffic received by all wireless nodes of ad hoc network during simulation time is shown in the following figure. The traffic received individually by destination node is less than source node. The high traffic received by nodes occur in selected route are mobile node 2 and intermediate node 2. As discussed above that intermediate nodes have high routing traffic because of Hello messages. During simulation time these nodes receive more than $600 \mathrm{bits} / \mathrm{sec}$ traffic in the whole scenario.

According to maximum routing traffic sent by DSR routing protocol in simulated scenario is between 2200 and 2400 bits/s, where 7 packets/s during communication. This is very good result during start of data packet transmission, but the routing traffic during transmission is draft to a very less value after the 20 seconds of route establishment. The routing traffic is reached to 0 bits and packets/s during communication and mostly less than 400 bits/s and 1 packet/s in the whole 300s simulation time. In the defined DSR routing protocol simulation scenario, maximum request period is keep to 10 seconds for route discovery. The initial interval between route discovery attempts is defined 5 seconds. Interval between successive route discoveries becomes double after each attempt for target connection. The process is repeated until a valid RREP is received for desired target. The total delay occurs in DSR protocol data transmission during simulation period. The delay is a bit high in start of the route establishment process, which is 0.0065 per second. The graph shows that delay becomes suddenly high and low during whole simulation period. The DSR delay is low because there is no Hello messages to send by nodes and routing traffic wait for that. MANET delay of DSR routing protocol is very low as compared to AODV routing protocol. Total DSR Traffic sent and received in simulated scenario for transmission of data between source and 
destination nodes consists of four nodes, i.e. source, mobile node 2 , intermediate node 2 , and destination. Source node sent traffic only once during start of transmission. During reply by destination node a lot of traffic is added to the data as shown in the figure. The intermediate node 2 and mobile node 2 ads nearly same data traffic during simulation time. The source node sent less than $100 \mathrm{bits} / \mathrm{sec}$ traffic where a mobile and intermediate node 2 sent more than 500 bits/sec traffic as shown in figure 3 and figure 4 respectively.

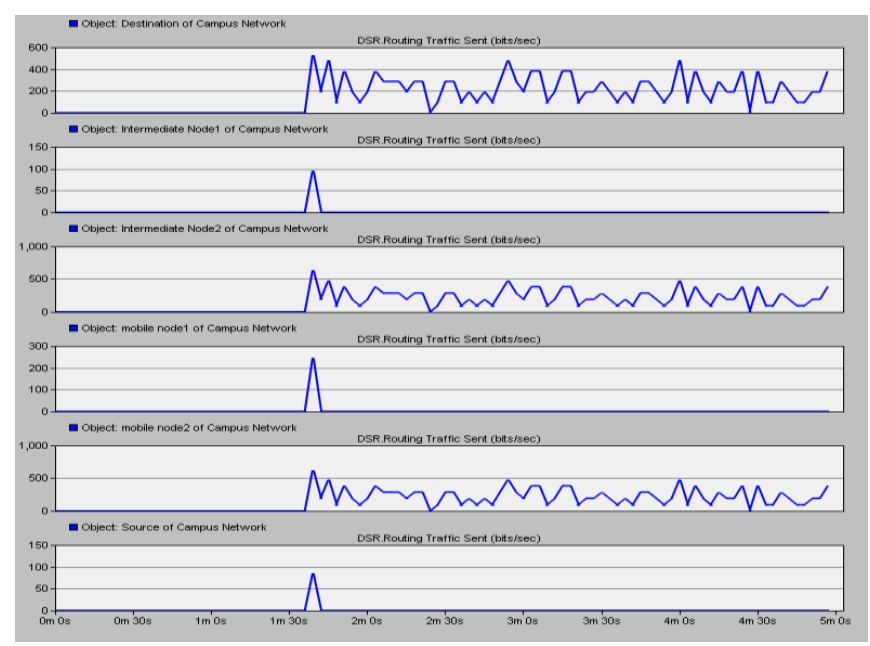

Figure 3. Total DSR routing traffic sent.

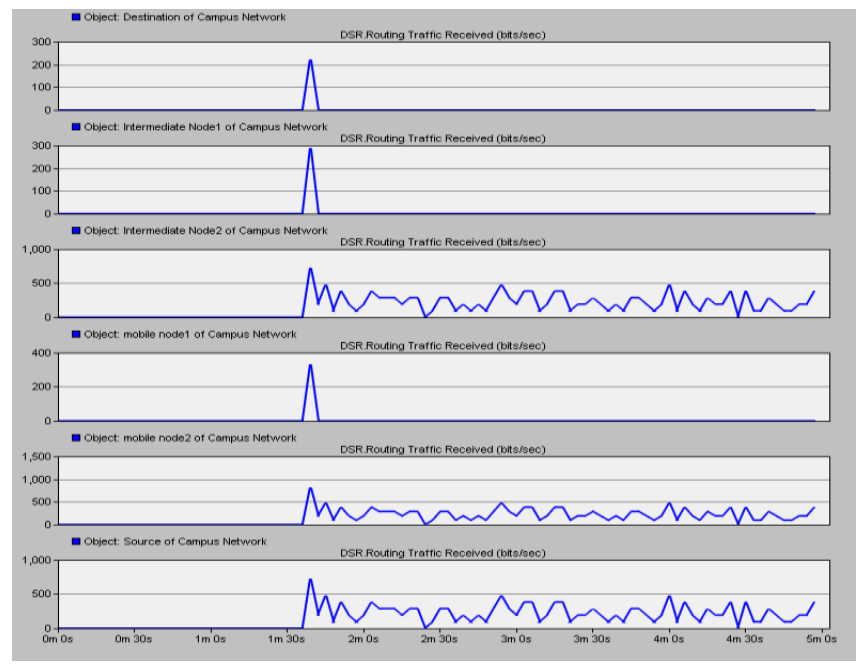

Figure 4. Total DSR routing traffic received

As a result DSR deliver great percentage of originated data packets where there is great mobility occurs. Fraction of data packets as a function of both mobility rate and network load for each protocol to deliver. An approach to providing QOS using MANET protocols based on best ratio of data packets from source to destination in an autonomous system..

\section{CONCLUSION}

MANET uses time to live (TTL) to avoid such loops, but more structured approach is required to get best results. The MANET delay effects on routing very badly, as the mobile nodes are battery powered and scarce memory. The routing protocol needs to be routed intelligently, utilize network bandwidth and energy resources in a better way in case of network route delay. The simulated scenario shows that routing overhead for the routing protocols heavily depends on node mobility. AODV sends many small routing control packets, while DSR sends less but bigger control packets during transmission of data packets. DSR is more useful in smaller networks with less mobility and usage of AODV more appropriate in ad hoc networks with a higher mobility and data transfer rate. The simulations results and analysis shows that mobile ad hoc network definitely needs more precise routing protocols to support increase mobility. I came to the conclusion that for small network DSR is suitable and for medium network AODV is appreciate able, in order to extend autonomous system to hybrid networks either for best QOS or VOIP.

\section{ACKNOWLEDGMENTS}

First of all I wanna thanks with core of my heart to IJCA for giving me the opportunity to express our ideas with research oriented world and I also wanna thank my family for their support and pray.

\section{REFERENCES}

[1] Halgamuge, K. S. \& Wang, P. L. (2005) Classification and Clustering for Knowledge Discovery. $1^{\text {st }}$ edition. Netherlands, Springer.

[2] Jahangir khan, "Base on MANET the correctness and performance evaluation of DSR,AODV and TORA Routing protocols for best QOS "International Journal of Computer Applications, Foundation of Computer Science, New York, USA. http://www.ijcaonline.org/specialissues/manets/number3/10 29-74.

[3] Jahangir khan, "Handover management in GSM cellular system". International Journal of Computer Applications, Foundation of Computer Science, New York, USA. http://www.ijcaonline.org/archives/volume8/number12/12571763

[4] Kil Sup Lee, Sungjong Lee, Yeon Ki Chung (2005), A performance comparison of on-demand Routing protocols for application data in Mobile ad-hoc networks.(SERA'05) IEEE.

[5] Lang, D. (2006) On the Evaluation and Classification of Routing Protocols for Mobile Ad Hoc Networks. [Internet].

$<$ http://home.leo.org/ dl/dissertation.pdf >

[6] Lee, S. (2000) Routing and Multicasting Strategies in Wireless Mobile Ad Hoc Networks: <http://www.sigmobile.org/phd/2000/theses/sjlee.pdf>

[7] Mukija, A. (2001) Reactive Routing Protocols for Mobile Ad Hocnetwork.<http://www.ifi.unizh.ch/ mukhija/papers/rrp_thesis. pdf>

[8] Shrirang.Ambaji.Kulkarni, Dr.G Raghavendra Rao(2008), Mobility Model Perspective for Scalability and Routing Protocol Performances in Wireless Ad-Hoc Networks. First international Conference on Emerging trends in Engineering and Technology.IEEE (2008).

[9] Suresh, A. (2005) Performance Analysis of Ad Hoc Ondemand Distance Vector routing (AODV) using OPNET Simulator. [Internet]. University of Bremen.Available from: http://www.comnets.uni-bremen.de/ koo/OPNET-AODV-asn.pdf 